Sharif University of Technology
Scientia Iranica

\title{
Designing and analyzing two non-invasive current sensors using Ampere's force law
}

\author{
M.R. Zamani Kouhpanji* \\ Department of Electrical and Computer Engineering, University of Minnesota Twin Cities, Minneapolis, MN 55455, USA.
}

Received 18 January 2019; received in revised form 15 May 2019; accepted 16 November 2019

\author{
KEYWORDS \\ Non-invasive current \\ sensors; \\ Low power \\ consumption; \\ High sensitivity; \\ Dynamic response; \\ Piezoelectric \\ micro-beam; \\ Non-magnetic \\ component.
}

\begin{abstract}
In this study, two different non-invasive current sensors are proposed, modeled, and analyzed. The current sensors are based on Ampere Force Law (AFL), representing the magnetic force between two parallel wire-carrying currents. These current sensors can be used for detecting/sensing DC and AC currents as well as their combination in a single wire or multiple wires. One of the most advantageous of them is that they do not rely on any permanent magnets for operation. In the first configuration, there are two microbeams, one of which is in the vicinity of the wire and undergoes mechanical vibrations due to the magnetic force between the wire and the microbeam. The movement of the microbeam, generating a magnetic field, induces a current inside another microbeam, which is stationary, as the output signal of the current sensor. In the second configuration, a single composite piezoelectric microbeam is used. The magnetic force between the wire and the piezoelectric microbeam forces the piezoelectric microbeam to move, thus producing a voltage. Both configurations present extremely low power consumption, which is not dependent on the sensitivity of the current sensors. The dynamic response, sensitivity, and power consumption of the current sensors are investigated, compared, and discussed.
\end{abstract}

(C) 2022 Sharif University of Technology. All rights reserved.

\section{Introduction}

Nowadays, the uncountable benefits of quantum effects and size dependency phenomena have provided a great motivation for scientists to miniaturize electronic and photonic devices. This trend has made nanotechnologies the most dominant research field on a global scale. However, these miniaturized electronic and photonic nanodevices require highly accurate nano/microelectromechanical devices (NEMS/MEMS) such as current sensors to precisely control their power to reach their maximum quantum efficiency [1-7].

\footnotetext{
*. Tel.: +1(618)-305-9636

E-mail address: zaman022@umn.edu
}

doi: $10.24200 /$ sci. 2019.52725 .2858
Moreover, it should be emphasized that the applications of the current sensors are not limited only to nanotechnologies. They are also a fundamental part of power networks, where any over/under flowing current damages the whole network [8].

A larger number of relevant research findings on the current sensors were mainly manifested in early studies of Edwin Hall in 1879 [9], followed by several intellectual non-invasive current sensors such as magnetoresistive sensor [10], Rogowski coil sensors $[11,12]$, and Hall effect sensors [13,14]. The novelty of the non-invasive current sensors is that they can measure the current flowing inside a wire without the need for being inserted in the circuit as it used to be the case for traditional current sensors.

This advantage of the non-invasive current sensors makes them an excellent candidate for health monitor- 
ing and diagnosis of electrical circuits, ranging from industrial and household scales to portable electronic devices such as cellphones. For diagnosis of industrial equipment, the current sensor can be easily placed next to wires to measure the flowing current without the need for cutting the wire. This feature reduces diagnostic time and facilitates the process. For household applications, the current sensor can be used to monitor the electrical consumption for controlling power consumption at peak times. Or, if there is an electrical discharge, the current flow suddenly changes. Thus, the current sensor can shut off the equipment for safety reasons. For portable electronic devices, these types of current sensors can monitor power consumption with different applications. In case when the device is running out of battery, the current sensor can give notifications to the user to close the application for saving battery. In addition to the high power consumption of traditional current sensors, they need to encircle wires to sense the current. This practical limitation causes these current sensors not to be used when there is more than one current in the nanodevice or network. Attempts to improve the performance of the current sensor result in the development of a new design of current sensors such as magnetic tunnel junction $[15,16]$, piezoresistive sensors [17], and delta-f resonant sensors $[18,19]$. In a more realistic evaluation of these current sensors, they have a very low power consumption rate in some cases and there is always a tradeoff among the power consumption, the sensor's sensitivity, and the magnetic field saturation of these current sensors [9].

To avoid the tradeoff, a new family of the current sensors has emerged which does not require any power supply [10-21]. This family consists of a permanent magnet and a piezoelectric microbeam, where the former is fixed at the tip of the piezoelectric microbeam. In these current sensors, the interaction between the permanent magnet and the magnetic field of the wire results in a mechanical force on the piezoelectric microbeam. The main drawback of these current sensors is the low durability and reliability of the sensors. This is the reason why the characterization and performance of the sensors highly depend on the magnetic properties of the permanent magnet [22] and the piezoelectric properties of the microbeam that degenerate over time [23-25]. Furthermore, this type of current sensors is mainly used for paired wire configurations, where there is a uniform magnetic field gradient. In the case of a single wire, not only is the magnetic field gradient non-uniform but it is also nonlinear in the space surrounding the wires, where the position and orientation of the current sensor must be precisely determined $[10,23,26,27]$ to ensure decent accuracy and sensitivity.

Consequently, the first motivation of this work is to design and analyze two alternative non-invasive current sensors without any limitations on the number of wires carrying unknown currents. The second motivation is to enhance the performance and reliability of the current sensors by avoiding uncertainties due to the properties of the materials used in the current sensor. To do so, two different current sensor configurations are proposed, designed, and analyzed. In both configurations, instead of using a permanent magnet, a microbeam carrying a current was used to interact with the magnetic field of the wire, which caused the microbeam to vibrate. Then, the mechanical vibration is converted to a voltage as the sensors' output.

\section{Modeling}

In this section, the two alternative configurations of the non-contact current sensors are presented. Figure 1(a) shows the current sensor based on a parallel beam carrying current, and Figure 1(b) shows the current sensor based on a single piezoelectric beam. For Figure 1(a), as the first configuration, the current sensor consists of two microbeams that can be made of any conductive materials, while for Figure 1(b), as the second configuration, the current sensor has a layer of a piezoelectric material on top and bottom separated by a shim layer. It is noted that the shim is usually chosen to be a metallic layer to help extract the current produced by piezoelectric layers and reduce the equivalent stiffness of the cantilever. To ensure the simplicity of the formula, it is assumed that the wire is very long and its cross-section is much smaller than those of the microbeams. However, the effects of the wire's dimensions can be readily considered [23,28,29]. Thus, the magnetic field around a wire carrying current can be obtained as follows:

$$
\vec{H}=\frac{i_{w}}{2 \pi r}\left(\cos (\alpha) \hat{e}_{z}-\sin (\alpha) \hat{e}_{y}\right)
$$

where $i_{w}$ is the current inside the wire which is unknown, and it can be either $\mathrm{DC}$ or $\mathrm{AC} ; e_{z}$ is the unity vector along $z$-axis; $e_{y}$ is the unity vector along $y$-axis; $r$ is the distance of each point in the space from the wire's center; see Figure 2. For convenience, each configuration is analyzed separately in designated sections as follows. Furthermore, all microbeams are along $x$-axis, and $y-z$ plan is perpendicular to the microbeams axis.

\subsection{The first configuration}

Using Eq. (1) and the parameters presented in Figure 1, the total force applied on the moving microbeam can be determined based on AFL as follows:

$$
\vec{F}=\mu_{0} L \int_{-h_{/ 2}}^{h_{/ 2}} \int_{d_{w m}}^{d_{w m}+t}\left(\vec{i}_{m} \times \vec{H}\right) d y d z
$$




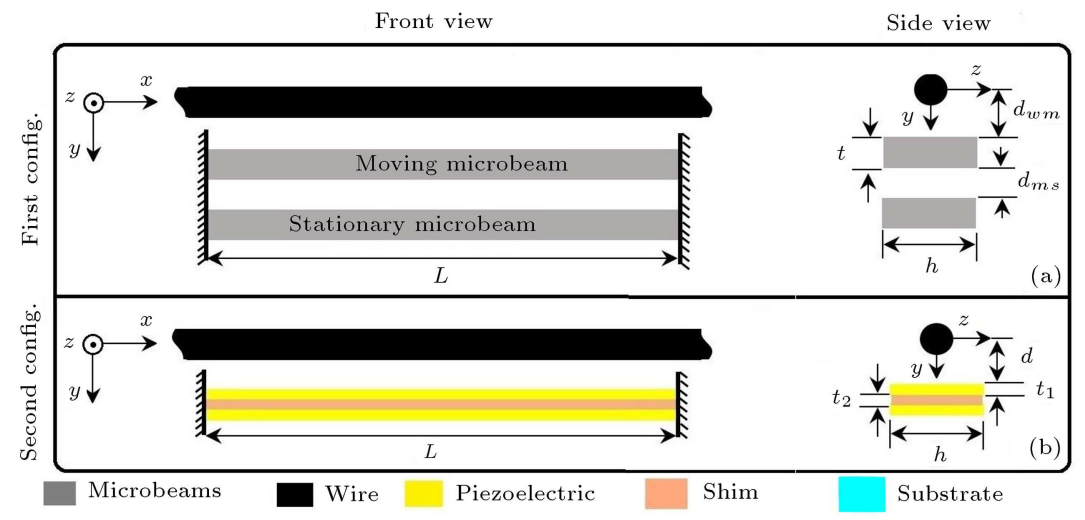

(a) A schematic 2D image of the current sensors, including the dimensions and side views.

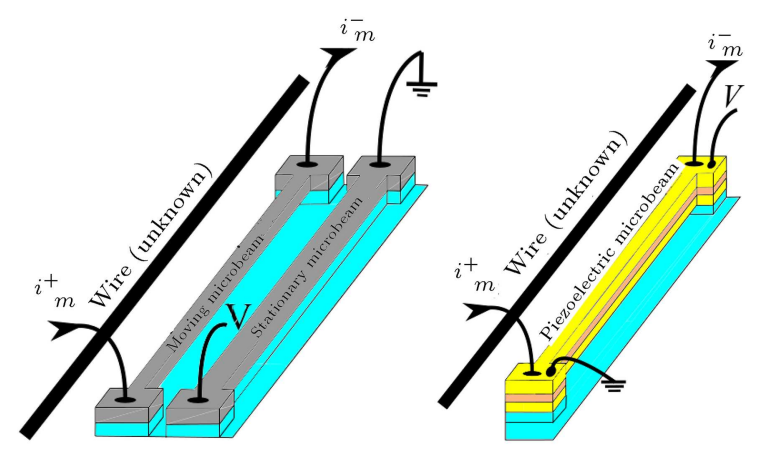

(b) A schematic 3D image of the current sensors. The left image shows the 1st configuration and the right image shows the 2nd configuration.

Figure 1. The front view and side view of the proposed configurations for the current sensors. In the first configuration, the microbeams have the same cross-section area. For the second configuration, the two piezoelectric layers have the same cross-section dimensions, and the total cross-section area is equal to the area of the first configuration's microbeams.

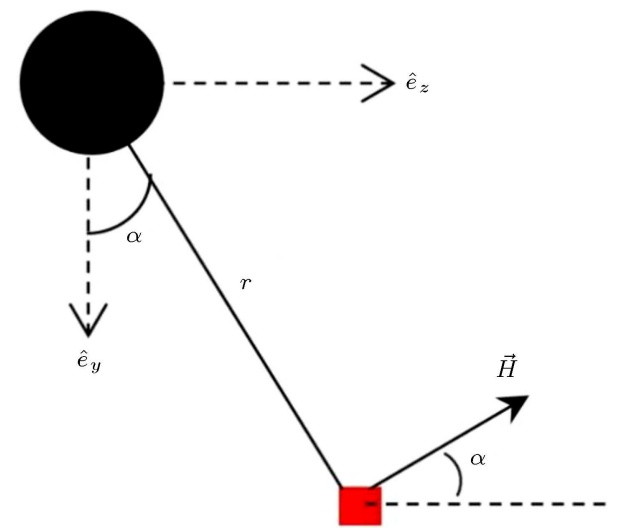

Figure 2. Magnetic field generated around the wire due to its current.

$$
=-\mu \frac{i_{w} i_{m}}{2 \pi} L \int_{-h_{/ 2}}^{h_{/ 2}} \int_{d_{w m}}^{d_{w m}+t}\left(\frac{y}{z^{2}+y^{2}} \hat{e}_{y}+\frac{z}{z^{2}+y^{2}} \hat{e}_{z}\right)
$$

$d y d z$

where $\mu_{0}$ is the permeability of the vacuum; $i_{m}$ is the current inside the microbeam; $t$ is the thickness of the moving microbeam; $h$ is the width of the moving microbeam; $L$ is the length of the microbeams; and $d_{w m}$ is the distance between the wire and the moving microbeam. Due to the symmetrical condition, the force in the $z$-direction is equal to zero. Moreover, as explained in the Electronic Supplementary Information (ESI), the integral in Eq. (2) has an analytical solution in which the total force applied to the microbeam can be determined as follows:

$$
\begin{aligned}
F= & \mu_{0} \frac{i_{w} i_{m}}{2 \pi}\left[\frac{h}{2} \ln \left(\frac{h^{2}+4\left(d_{w m}+t\right)^{2}}{h^{2}+4 d_{w m}^{2}}\right)\right. \\
& -2 d_{w m} \tan ^{-1}\left(\frac{h}{2 d_{w m}}\right)+2\left(d_{w m}+t\right) \\
& \left.\tan ^{-1}\left(\frac{h}{2\left(d_{w m}+t\right)}\right)\right] L .
\end{aligned}
$$

Of note, the total force applied on the microbeams remains in the y-direction, and its direction and frequency are dependent on the product of the currents flowing in the wire and the microbeams, moving microbeam for the 1 st configuration and the piezoelectric microbeam for the 2 nd configuration. 
The next step is to model the dynamic response of the moving microbeam. The moving microbeam can be modeled as a simple mass connected to a spring and damper. Therefore, the equation of motion of the moving microbeam can be written as follows:

$$
M \ddot{w}+\lambda \dot{w}+k w=F,
$$

where $M$ is the moving microbeam's mass; $\lambda$ is the damping coefficient; $k$ is the spring constant of the moving microbeam equal to $32 E h t^{3} / L^{3}$; and $E$ is Young's modulus of the moving microbeam. Furthermore, $w$ is the displacement of the center of the moving microbeam, and the dots represent the time derivative of the displacement. Thus, the transfer function of the moving microbeam is:

$$
W(s)=\frac{F}{M} \frac{1}{s^{2}+2 \zeta \omega_{n} s+\omega_{n}^{2}},
$$

where $\zeta$ is the damping ratio equal to $\lambda / 2 \sqrt{k M}$; and $\omega_{n}$ is the natural frequency of the moving microbeam equal to $\sqrt{k / M}$. It should be mentioned that, according to [30], the displacement of each point along the moving microbeam can be determined by multiplying its time response (determined by solving Eq. (4)) and the eigenfunction of the moving microbeam. The eigenfunction of a continuous microbeam under a fixedfixed boundary condition, $\psi(x)$, was given [30]:

$$
\begin{aligned}
\psi(x)= & \cosh \left(\gamma_{n} \frac{x}{L}\right)-\cos \left(\gamma_{n} \frac{x}{L}\right) \\
& -\frac{\cosh \left(\gamma_{n}\right)-\cos \left(\gamma_{n}\right)}{\sinh \left(\gamma_{n}\right)-\sin \left(\gamma_{n}\right)}\left(\sinh \left(\gamma_{n} \frac{x}{L}\right)\right. \\
& \left.-\sin \left(\gamma_{n} \frac{x}{L}\right)\right)
\end{aligned}
$$

Here, $\gamma_{n}$ corresponds to the mode of resonant of the moving microbeam, which is the solution of $\cosh \left(\gamma_{n}\right) \cos \left(\gamma_{n}\right)=1$.

Once the moving microbeam starts vibrating, it induces a current inside the stationary microbeam because of its relative velocity and the magnetic field generated by its current [31]. The voltage induced between the two ends of the stationary microbeam can be determined as follows:

$$
\begin{aligned}
\Delta V_{m}= & \mu \int_{0}^{L}(\overrightarrow{\dot{w}} \times \vec{H}) d x=\frac{1}{\psi\left(\frac{L}{2}\right) A_{s}} \int_{0}^{L} \psi(x) d x \\
& \left(\int_{h_{/ 2}}^{h_{/ 2}} \int_{d_{m s}+t}^{d_{m s}+2 t} \dot{w} H_{z}\left(v_{0}, u_{0}\right) d v_{0} d u_{0}\right),
\end{aligned}
$$

where $\dot{w}$ is the velocity at the center of the moving microbeam; $A_{s}$ is the cross-section area of the stationary microbeam; $d_{m s}$ is the distance between the moving microbeam and stationary microbeam; and $H_{z}$ is the magnetic field generated by the moving microbeam in the $z$-direction determined by Feynman et al. [32]:

$$
\begin{gathered}
H_{x}\left(v_{0}, u_{0}\right)=\frac{i_{m}}{2 \pi A_{m}} \int_{-h_{/ 2}}^{h_{/ 2}} \int_{0}^{t} \frac{v_{0}-v}{\left(u_{0}-u\right)^{2}+\left(v_{0}-v\right)^{2}} \\
d v d u,
\end{gathered}
$$

where $A_{m}$ is the cross-section area of the moving microbeam, and $u-v$ are the axes of the coordinate system located at the center of the upper face of the moving microbeam while the $x$-axis is along the moving microbeam's axial axis. Moreover, $(u, v)$ and $\left(u_{0}, v_{0}\right)$ represent points on the moving and stationary microbeams, respectively, in this coordinate system.

It is noteworthy that the frequency of the output signal in this configuration is a combination of the frequencies of the wire's current and the moving microbeam's current because the output signal is the result of the products of the currents that have sinusoidal terms according to their frequency. However, if the wire's current is a DC current, the output signal frequency is only dependent on the frequency of the microbeam's current, and it has the following timedependent term:

$$
\frac{d}{d t}\left(\sin \left(\omega_{m} t\right)\right) \sin \left(\omega_{m} t\right)=\frac{\omega_{m}}{2} \sin \left(2 \omega_{m} t\right) .
$$

If the wire has an $\mathrm{AC}$ current, then the output signal has the following time-dependent term (left side of Eq. (10) which can be simplified to summation as the right side of the following equation:

$$
\begin{aligned}
& \frac{d}{d t}\left(\sin \left(\omega_{w} t\right) \sin \left(\omega_{m} t\right)\right) \sin \left(\omega_{m} t\right)=\frac{\omega_{w}}{2} \cos \left(\omega_{w} t\right) \\
& \quad-\frac{\omega_{w}+\omega_{m}}{4} \cos \left(\left(\omega_{w}+2 \omega_{m}\right) t\right)-\frac{\omega_{w} \omega_{m}}{4} \\
& -\cos \left(\left(\omega_{w}-2 \omega_{m}\right) t\right),
\end{aligned}
$$

where $t$ is time, $\omega_{w}$ the frequency of the wire's current, and $\omega_{m}$ the frequency of the moving microbeam's current.

\subsection{The second configuration}

The force applied to each layer of the piezoelectric microbeam can be calculated using Eq. (3), and the total force applied to the piezoelectric microbeam is the summation of all forces. Note that the total current flowing in the piezoelectric microbeam is the summation of all currents flowing in each layer. To determine each layer current, the piezoelectric microbeam can be modeled as three parallel resistances. Furthermore, 
it should be mentioned that the force applied to the piezoelectric microbeam is mainly due to the input current, not the current induced inside it by mechanical vibration. This is the reason why the induced current is usually smaller than the input current by several orders of magnitude.

Modeling the dynamical vibration of the piezoelectric microbeam is completely different from a regular microbeam such as the 1st configuration. First, the piezoelectric microbeam is made of three layers where each layer can have different mechanical properties and thicknesses. Therefore, the equivalent stiffness, $(E I)_{e q}$, of the microbeam must be considered to take into account the variations of the properties of the materials and sizes on the mechanical stiffness. The equivalent mechanical stiffness can be determined using:

$$
\begin{aligned}
(E I)_{e q} & =\sum_{j=1}^{3} E_{j} I_{j}=2 E_{P Z} \\
& \left(\frac{1}{12} h t_{1}^{3}+t_{1} h\left(\frac{t_{1}}{2}+\frac{t_{2}}{2}\right)^{2}\right)+\frac{1}{12} E_{d} h t_{2}^{3},
\end{aligned}
$$

where $t_{1}$ is the thickness of the piezoelectric layers; $h$ the width of the piezoelectric layers which is equal to the shim layer width; $t_{2}$ is the thickness of the shim layer; $E_{P Z}$ Young's modulus of the piezoelectric layers; and $E_{d}$ Young's modulus of the shim layer.

Second, because the existence of the charges on the piezoelectric layers impacts the mechanical behavior of the piezoelectric, the governing equations of motion for the piezoelectric microbeam are electromechanically coupled. The governing equations of motion for piezoelectric microbeams under forced vibration were derived from [33] using analogy within the electrical circuit and mechanical structures. Roundy and Wright [33] derived the equation of motion of a piezoelectric microbeam as follows:

$$
\begin{aligned}
& \ddot{S}+2 \zeta \omega_{n} \dot{S}+\omega_{n}^{2} S=\frac{k_{s p} a_{1} d_{31}}{M_{e q}} V+\frac{F}{k_{2} M_{e q}}, \\
& \dot{V}=\frac{a_{3} c_{p} d_{31} t_{1}}{a_{2} \varepsilon} \dot{S}
\end{aligned}
$$

where $S$ is the axial strain inside the piezoelectric layers and the dots again show the time derivative of the axial strain; $V$ is the voltage between the two ends of the piezoelectric microbeam; $M_{e q}$ is the equivalent mass of the piezoelectric microbeam which is equal to the mass summation of all layers; $d_{31}$ is the coefficient of the piezoelectric layers; $c_{p}$ is the capacitance induced between the piezoelectric layers due to accumulation of charges; $a_{1}, a_{2}$, and $a_{3}$ are the coefficients related to the configurations between the piezoelectric layers; $k_{s p}$ is the spring constant of the piezoelectric microbeam equal to $384(E I)_{e q} / L^{3}$.
Lastly, $k_{2}$ is a geometric coefficient which is dependent on the boundary condition, dimension, and length of the piezoelectric microbeam at boundaries $L_{e}$. The $k_{2}$ for a fixed-fixed boundary condition is equal to:

$$
k_{2}=\frac{L^{4}}{32 t_{1}} \frac{1}{2 L^{2}-3 L L_{e}+L_{e}^{2}} .
$$

By solving Eq. (12), the AC voltage induced between the two ends of the piezoelectric microbeam can be achieved as follows [10]:

$$
\Delta V_{p z}=\frac{1}{s^{2}+2 \zeta \omega_{n} s+\omega_{n}^{2}\left(1-\frac{d_{31} c_{p}}{\varepsilon}\right)} \frac{d_{31} c_{p} t_{1} a_{3}}{k_{2} M_{e q} \varepsilon a_{2}} F,
$$

where $\varepsilon$ is the dielectric constant of the piezoelectric layers.

The force applied to the microbeam in the $2 \mathrm{nd}$ configuration results from the interaction between the magnetic field induced by the current of the wire and the microbeam. According to Eq. (3), this force is the product of both currents. If the wire carries an $\mathrm{AC}$ current, its output signal will have the product of the sinusoidal functions as $\sin \left(\omega_{w} t\right) \sin \left(\omega_{p z} t\right)$, which can be readily simplified as follows:

$$
\begin{aligned}
& \sin \left(\omega_{w} t\right) \sin \left(\omega_{p z} t\right)=\frac{1}{2} \cos \left(\left(\omega_{w}-\omega_{p z}\right) t\right) \\
& -\frac{1}{2} \cos \left(\left(\omega_{w}+\omega_{p z}\right) t\right)
\end{aligned}
$$

where $\omega_{p z}$ is the frequency of the piezoelectric microbeam current. Moreover, if the wire has a DC current, then the frequency of the output signal is equal to that of the piezoelectric microbeam's current. If the frequency of the wire is known, the frequency of the microbeam must be selected as in $\omega_{m}=\omega_{w}-\omega_{n}$ or $\omega_{m}=\omega_{n}-\omega_{w}$, where $\omega_{n}$ is the resonance frequency of the microbeam. In this case, the force applied to the microbeam will have at least one component at the resonance frequency of the microbeam, leading to the largest mechanical vibration amplitude for the microbeam and, consequently, the highest signal-tonoise ratio at the output. In practical applications, if the frequency of the wire is unknown, the user must conduct a sweep mode frequency measurement to see the response of the current sensor as it must be done for all current sensors. Once a sweep mode frequency measurement is conducted, there will be an output response associated with each of these frequencies. Then, using the provided formulas, one can easily determine the frequency of the wire, even if it is unknown, as well as the required frequency for the microbeam for further detections. A similar argument is valid for the 1st current sensor configuration. 


\section{Results and discussions}

The properties of the materials were taken from [34] for the piezoelectric layers and from [35] for the silicon microbeams and aluminum shim. Table 1 provides the physical parameters used for modeling and evaluating the designs.

Figures 3 and 4 demonstrate the dynamic responses of the current sensors by presenting the gain and phase shift of the current sensors. As can be seen, the maximum gains decrease as the damping ratio increases. In the proposed current sensors, the damping results from structural damping and viscous damping induced by the interaction between the microbeams and the air surrounding the current sensors, where structural damping has the dominant impact on the performance of the current sensors. Structural damp-

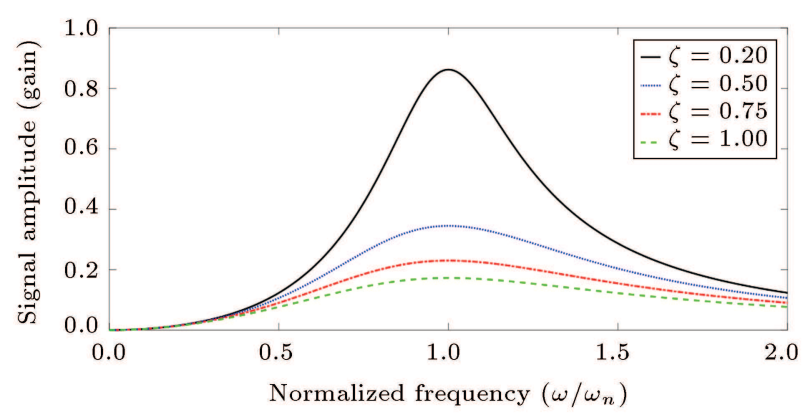

(a)

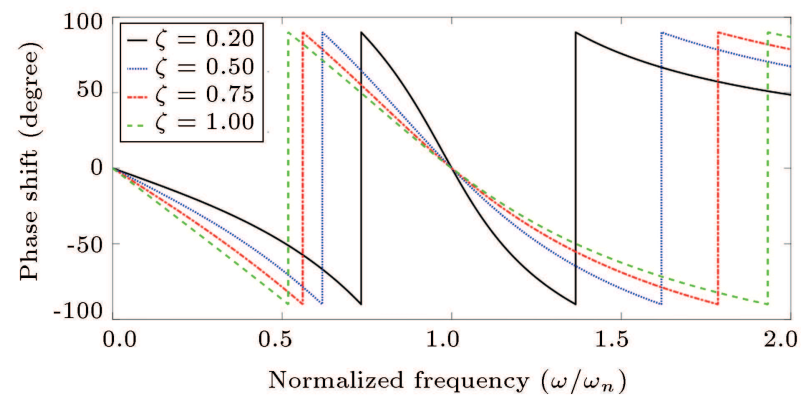

(b)

Figure 3. Dynamical response of the 1st current sensor: (a) Gain of the output voltage of the 1st current sensor, and (b) phase shift of the output voltage of the 1st current sensor. ing can be significantly reduced by either increasing the length of the microbeams or reducing the cross-section dimensions of the microbeams. Of note, the phase shifts of the two configurations are completely different from each other, even though their gains are almost the same. For the 1st configuration, the phase shift is equal to zero at the resonance frequency and it varies smoothly in the vicinity of the resonance frequency. However, for the 2nd configuration, the phase shift has a discontinuity in the resonance frequency and has a 180-degree phase shift.

It should be mentioned that the position of the maximum gain of the 1st current sensor is only dependent on the moving microbeam natural frequency and the damping ratio, while it depends on the piezoelectric coefficient, dielectric constant, and piezoelectric

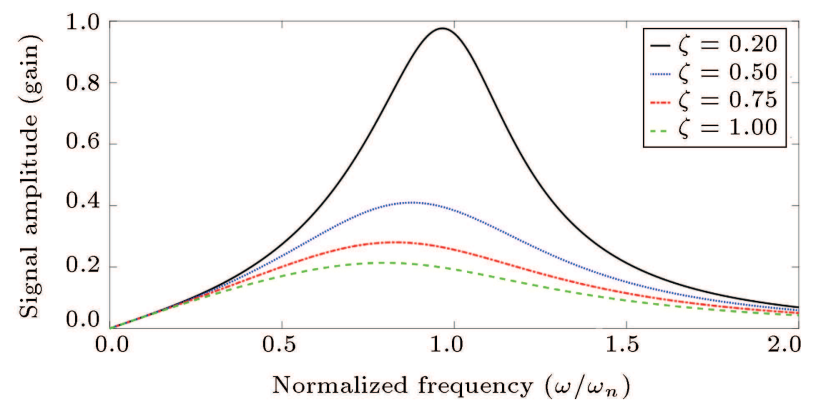

(a)

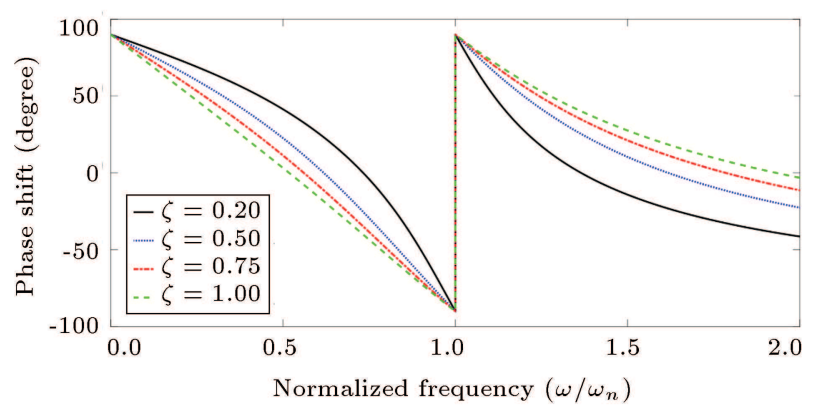

(b)

Figure 4. Dynamical response of the 2nd current sensor: (a) Gain of the output voltage of the 2nd current sensor, and (b) phase shift of the output voltage of the $2 \mathrm{nd}$ current sensor.

Table 1. Used parameters for modeling of the current sensors.

\begin{tabular}{|c|c|c|}
\hline Parameters & 1st configuration & 2nd configuration \\
\hline Thicknesses of the moving and stationary beams $(t)$ & $1 \mathrm{E}-6 \mathrm{~m}$ & - \\
\hline Thickness of the top piezoelectric later $\left(t_{1}\right)$ & - & $0.33 \mathrm{E}-6 \mathrm{~m}$ \\
\hline Thickness of the bottom piezoelectric layer $\left(t_{2}\right)$ & - & $0.33 \mathrm{E}-6 \mathrm{~m}$ \\
\hline Width of the microbeams $(h)$ & \multicolumn{2}{|c|}{$1 \mathrm{E}-6 \mathrm{~m}$} \\
\hline Length of the microbeams $(L)$ & \multicolumn{2}{|c|}{$1 \mathrm{E}-3 \mathrm{~m}$} \\
\hline Distance between the moving microbeam and the wire $\left(d_{w m}\right)$ & \multicolumn{2}{|c|}{$2 \mathrm{E}-6 \mathrm{~m}$} \\
\hline Distance between the stationary microbeam and the wire $\left(d_{m s}\right)$ & $2 \mathrm{E}-6 \mathrm{~m}$ & - \\
\hline
\end{tabular}




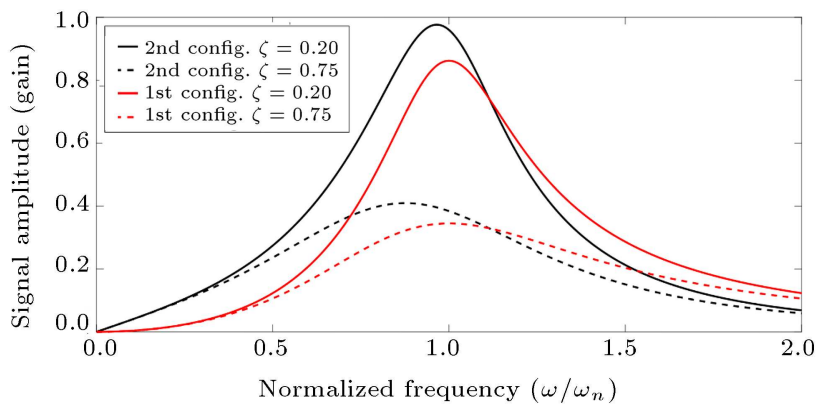

Figure 5. Comparison in the gain of the current sensors.

capacitance, in addition to the natural frequency and damping ratio of the 2 nd current sensor (see Eq. (14)). This matter justifies the dependence of the position of the maximum gain of the 2nd current sensor on the damping ratio which can be seen as a small shift in the position of the maximum gain of the 2 nd current sensor compared to the 1st current sensor (see Figure 5).

One of the critical factors in designing any sensor is the real-time sensitivity of the sensor. The real-time sensitivity is defined as a derivative of the output signal to the input signal of the sensor. According to Eqs. (7) and (14), the output signal in both current sensors is linearly proportional to the input currents, which is one of the superior advantages of these configurations compared to the prior current sensors. This linear behavior of the current sensors makes the real-time sensitivity of the current sensors the same as their time responses without any dependency on the wire frequency, wire current amplitude, or physical parameters of the wire. This superior advantage of these current sensors makes it possible to optimize an initial design of these current sensors. Then, it can be used for any input signal with the same performance. Therefore, real-time sensitivity of the proposed current sensors is as follows:

$$
\text { Sensitivity } \equiv \frac{d(\Delta V)}{d I},
$$

where $\Delta V$ is the output voltage of the current sensors and $I$ is the product of the amplitudes of the unknown current inside the wire and the current of the microbeams. Figure 6 depicts the time response of the current sensors for a sinusoidal input signal. In Figure 6, all physical parameters are assumed to be the same for both current sensors, in which the total thickness of the piezoelectric microbeam is the same as the thickness of the moving and/or stationary microbeams. The initial parts of the time response, equivalent to the real-time sensitivities, of the current sensors are affected by the transient response of the sensors, and they reach steady-state conditions after a few cycles. It should be emphasized that the 1st configuration provides a faster dynamic response than the 2 nd configuration under the same conditions.

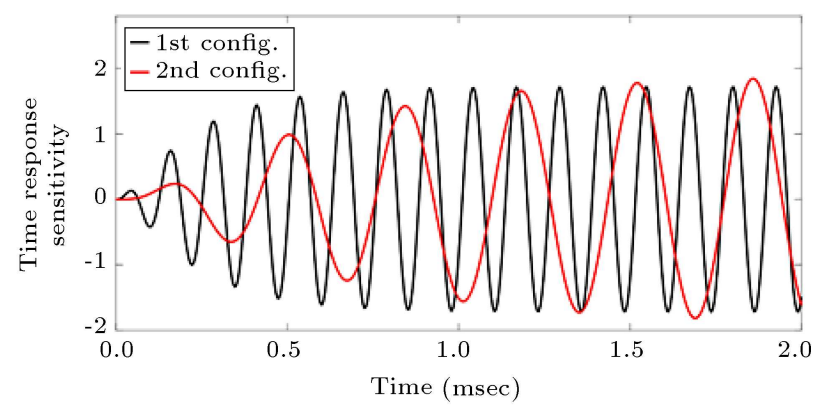

Figure 6. Time response of the current sensors for a sinusoidal input current with $\zeta=0.5$; the frequency of the wire and microbeam is assumed to be equal to the half of the resonance frequency of the microbeam.

The power consumption of the current sensors is also analyzed, which is another critical factor in designing highly efficient current sensors. In both configurations, the input power is related to the power consumed in the microbeams for generating the mechanical vibration. Therefore, we have:

1st configuration: $\quad P_{i n}=i_{m}^{2} R_{m}$

2nd configuration: $\quad P_{i n}=i_{p z}^{2} R_{p z}$,

where $i_{p z}$ is the input current in the piezoelectric microbeam; $R_{m}$ is the electrical resistance of the moving microbeam; and $R_{p z}$ is the electrical resistance of the piezoelectric microbeam. Another superior advantage of these configurations compared to previous current sensors is that the consumed power is only dependent on the electrical resistance of the microbeams and the input current. On the other hand, according to Eqs. (7) and (14), since the output signal is independent of the electrical resistance of the microbeams, the power consumption can be minimized to zero by increasing the doping level into the microbeams or using a metallic moving microbeam for the 1 st configuration.

To investigate the power efficiency, $P_{\text {out }} / P_{\text {in }}$, of the current sensors, here the output power of the current sensors is calculated. Since the output signal is a voltage, the output power is:

$$
\begin{array}{ll}
\text { 1st configuration: } & P_{\text {out }}=\frac{\Delta V_{m}^{2}}{R_{s}}, \\
\text { 2nd configuration: } & P_{\text {out }}=\frac{\Delta V_{p z}^{2}}{R_{p z}},
\end{array}
$$

where $R_{s}$ is the electrical resistance of the stationary microbeam which is the same as $R_{m}$. Figure 7 shows the normalized power efficiency of the current sensors.

According to Figure 7, the power efficiency of the current sensors is the same for the same physical parameters, and it only depends on the damping ratio of the sensors. Of note, even though the power efficiency 
Table 2. Qualitative comparison between the two configurations.

\begin{tabular}{ccc}
\hline Practical concern & 1st configuration & 2nd configuration \\
\hline Sensitivity & Good & Medium \\
Power consumption & Good & Good \\
Signal to noise ratio & Medium & Good \\
Linearity in response & Good & Medium \\
Fabrication process & Medium & Complicated \\
\hline
\end{tabular}

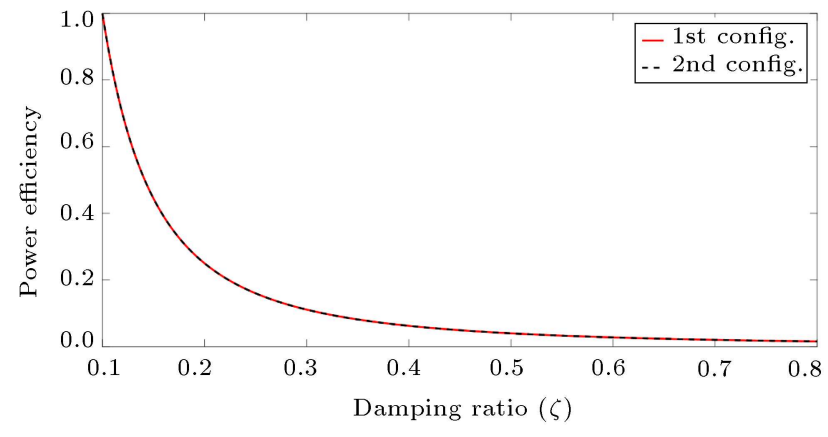

Figure 7. Power efficiency of the current sensors normalized to the power efficiency at $\zeta=0.10$. For simplicity, it is assumed that the wire carries a DC current and the microbeams carry a current at their resonance frequency.

of the current sensors is the same, their output power could be different due to different rates of electrical resistance of the microbeams (see Eq. (18)). Table 2 is provided to summarize the novelty of the proposed current in a nutshell. For the sake of comparison, it is assumed that both configurations have the same dimensions.

\section{Conclusion}

Two different non-invasive current sensors were proposed, designed, and analyzed here based on the Ampere Force Law (AFL). The current sensors could detect/sense both DC, AC, and their combinations in a single wire or multi-wire network. For the same physical parameters, the 1st configuration (current sensor using two parallel microbeams) presents a faster dynamic response than the 2 nd configuration (current sensor using a piezoelectric microbeam). However, the 2nd configuration presents a higher gain than the 1st configuration. Furthermore, in terms of the experiment, the 2 nd configuration is more difficult to fabricate due to the required extra steps for implementing the piezoelectric material on the microbeam. The dynamic response and the sensitivities of the current sensors were linearly dependent on the wire current and wire frequency. The power consumption of the current sensors was examined and it was found that the power consumption of the current sensors was only dependent on the electrical resistance of the microbeams, which demonstrated extremely low power consumption for the current sensors. Furthermore, studying the power efficiency of the current sensors illustrated that there was not any tradeoff or dependency between the power consumption and the sensitivity and the dynamic response of the current sensors. Therefore, it is concluded that the sensitivity and dynamic response of the current sensors can be maximized without affecting the power efficiency of these current sensors. Furthermore, the dynamic response analysis, sensitivity analysis, and power consumption analysis of the proposed current sensors confirmed the practical realization of the proposed current sensors in both industrial and household applications.

\section{Supplementary information}

Supplementary information is available at: http://scientiairanica.sharif.edu/jufile?ar_sfile $=126734$

\section{References}

1. Cao, Xi, Xiao-Jian Tian, and Dong F. Wang "Wireless electric current sensing via integrating a magneticpiezoelectric cantilever with a microstrip antenna", Micro \& Nano Letters, 12(11), pp. 871-874 (2017).

2. Kouhpanji, M.R.Z., Behzadirad, M., Feezell, D., and Busani, T. "Insufficiency of the Young's modulus for illustrating the mechanical behavior of GaN nanowires", Nanotechnology, 29(20), p. 205706 (2018).

3. Zamani Kouhpanji, M.R. "Investigating the classical and non-classical mechanical properties of $\mathrm{GaN}$ nanowires", MS. Thesis, Univ. New Mex. (2017).

4. Xian, W., Li, X., Wang, D.F., Kobayashi, T., Itoh, T., and Maeda, R. "Precise current sensing using a piezoelectric cantilever based current sensor", Solid-State Sensors, Actuators Microsystems (TRANSDUCERS), 2017 19th Int. Conf., pp. 1057-1060 (2017).

5. Zamani Kouhpanji, M.R., Behzadirad, M., and Busani, T. "Classical continuum theory limits to determine the size-dependency of mechanical properties of GaN NWs", Journal of Applied Physics, 122(22), 225113 (2017).

6. Zamani Kouhpanji, M.R. and Jafaraghaei, U. "A semianalytical approach for determining the nonclassical mechanical properties of materials", J. Mech. Behav. Mater., 26(5-6), pp. 193-203 (2017). 
7. Alaie, S., Hossein-Zadeh, M., Baboly, M.G., Zamani, M., and Leseman, Z.C. "Enhancing mechanical quality factors of micro-toroidal optomechanical resonators using phononic crystals", J. Microelectromechanical Syst., 25(2), pp. 311-319 (2016).

8. Lao, S.B., Chauhan, S.S., Pollock, T.E., Schroder, T., Choi, I.S., and Salehian, A. "Design, fabrication and temperature sensitivity testing of a miniature piezoelectric-based sensor for current measurements", Actuators, 3, pp. 162-181 (2014).

9. Sherman, C.T., Wright, P.K., and White, R.M. "Sensors and Actuators A: Physical validation and testing of a MEMS piezoelectric permanent magnet current sensor with vibration canceling", Sensors Actuators A. Phys., 248, pp. 206-213 (2016).

10. Leland, E.S., White, R.M., and Wright, P.K. "Design and fabrication of a MEMS AC electric current sensor", Adv. Sci. Technol., 54, pp. 350-355 (2008).

11. Chattock, A.P. "On a magnetic potentiometer", The London, Edinburgh, and Dublin Philosophical Magazine and Journal of Science, 24(146), pp. 94-96 (1887).

12. Ward, D.A. and Exon, J.L.T. "Using Rogowski coils for transient current measurements", Eng. Sci. Educ. J., 2(3), p. 105 (1993).

13. Ramsden, E., Hall-Effect Sensors: Theory and Application, Newnes (2011).

14. Ziegler, S., Woodward, R.C., Iu, H.H.-C., and Borle, L.J. "Current sensing techniques: A review", IEEE Sens. J., 9(4), pp. 354-376 (2009).

15. Breth, L., Dimopoulos, T., Schotter, J., Rott, K., Bruckl, H., and Suess, D. "Fluxgate principle applied to a magnetic tunnel junction for weak magnetic field sensing", IEEE Trans. Magn., 47(6 PART 1), pp. 1549-1553 (2011).

16. Julliere, M. "Tunneling between ferromagnetic films", Phys. Lett. A, 54(3), pp. 225-226 (1975).

17. Lin, Q. Bin and Du, G.T. "The study of a MEMS magnetic field sensor based on 'cross-shape' ferromagnetic film", In Materials Science Forum, Trans Tech Publications Ltd, 694, pp. 523-527 (2011).

18. Hui, Y., Nan, T.X., Sun, N.X., and Rinaldi, M. "MEMS resonant magnetic field sensor based on an AlN/FeGaB bilayer nano-plate resonator", 2013 IEEE 26th Int. Conf. Micro Electro Mech. Syst., 1, pp. 721724 (2013).

19. Wang, D.F. and Maeda, R. "Analytical study on cantilever resonance type magnet-integrated sensor device for micro-magnetic field detection", Microsyst. Technol., 21(6), pp. 1167-1172 (2015).

20. Leland, E.S., White, R.M., and Wright, P.K. "Energy scavenging power sources for household electrical monitoring", PowerMEMS, pp. 165-168 (2006).

21. Wang, D.F., Liu, H., Li, X., Li, Y., Xian, W., Kobayashi, T., Itoh, T., and Maeda, R. "Passive MEMS DC electric current sensor: Part I-Theoretical considerations", IEEE Sensors Journal, 17(5), pp. 1230-1237 (2016).
22. Shang, X., Li, Y., Liu, H., Kobayashi, T., Wang, D.F., Itoh, T., and Maeda, R. "Developing MEMS DC electric current sensor for end-use monitoring of DC power supply: Part VI-Corresponding relationship between sensitivity and magnetic induction", Des. Test, Integr. Packag. MEMS/MOEMS (DTIP), Symp., pp. 1-4 (2017).

23. Zamani Kouhpanji, M.R. "Paired-wire carrying current actuators and piezoelectric beam sensors for microelectromechanical systems", Microsyst. Technol., 24(5), pp. 2401-2408 (2018).

24. Olszewski, O.Z., Houlihan, R., Blake, A., Mathewson, A., and Jackson, N. "Evaluation of vibrational piezoMEMS harvester that scavenges energy from a magnetic field surrounding an AC currentcarrying wire", Journal of Microelectromechanical System, 26(6), pp. 1298-1305 (2017).

25. Zamani Kouhpanji, M.R. "Demonstrating the effects of elastic support on power generation and storage capability of piezoelectric energy harvesting devices", J. Intell. Mater. Syst. Struct., 30(2), pp. 323-332 (2019).

26. Leland, E.S., Wright, P.K., and White, R.M. "A MEMS AC current sensor for residential and commercial electricity end-use monitoring", J. Micromechanics Microengineering, 19(9), p. 094018 (2009).

27. Xian, W. and Wang, D.F. "Position and orientation correction scheme for current sensing based on magnetic piezoelectric cantilevers", 143501, pp. 1-12 (2017).

28. Zamani Kouhpanji, M.R. "Studying the dynamical response of nano-microelectromechanical devices for nanomechanical testing of nanostructures", Int. J. Mech. Aerospace, Ind. Mechatron. Manuf. Eng., 11, pp. 1802-1809 (2017).

29. Zamani Koujpanji, M.R. "Designing and analyzing nano/microelectromechanical device for fatigue and fracture characterization of nanomaterials", Adv. Nat. Sci. Nanosci. Nanotechnol., pp. 351-1356-1, \#35 (2017).

30. Kahrobaiyan, M.H., Asghari, M., Hoore, M., and Ahmadian, M.T. "Nonlinear size-dependent forced vibrational behavior of microbeams based on a nonclassical continuum theory", J. Vib. Control, 18(5), pp. 696-711 (2012).

31. Nayfeh, M.H. and Brussel, M.K., Electricity and Magnetism, Courier Dover Publications (2015).

32. Feynman, R.P., Leighton, R.B., and Sands, M. "The Feynman lectures on physics", vol. I, American Journal of Physics, 33(9), pp. 750-752 (1965)

33. Roundy, S. and Wright, P.K. "A piezoelectric vibration based generator for wireless electronics", Smart Mater. Struct., 13(5), pp. 1131-1142 (2004).

34. Xiang, H.J. and Shi, Z.F. "Static analysis for functionally graded piezoelectric actuators or sensors under a combined electro-thermal load", Eur. J. Mech. A/Solids, 28(2), pp. 338-346 (2009). 
35. Rumble, J., CRC Handbook of Chemistry and Physics, CRC press (2017).

\section{Biography}

Mohammad Reza Zamani Kouhpanji is currently working as a Postdoctoral Research Scholar at the University of California Los Angeles. He started his educational journey from Mechanical Engineering by completing his BS and MS at Isfahan University of
Technology and Sharif University of Technology, Iran in 2011 and 2013, respectively. Afterward, he decided to pursue his career in Electrical Engineering, which he switch to it by starting his MS at the University of New Mexico followed by his PhD at the University of Minnesota. His diverse background helped him to be a successful researcher in the field of Nanobiotechnology by designing, fabricating, and characterizing advanced nanomaterials and NEMS/MEMS devices for a broad range of applications. 